\title{
APPLICATIONS OF NANOTECHNOLOGY IN INTERDISCIPLINARY DENTISTRY
}

\section{Dental Science}

Keerthivasan

Arumugam

\section{Vidhya Sampath*}

\section{Rajkumar Kothandaraman}

\section{Mahalaxmi Sekar}

Department of Conservative Dentistry and Endodontics, SRM Dental College, SRM Institute of Science and Technology, Ramapuram Campus, Bharathi Salai, Ramapuram, Chennai, TN, India-600089.

Department of Conservative Dentistry and Endodontics, SRM Dental College, SRM Institute of Science and Technology, Ramapuram Campus, Bharathi Salai, Ramapuram, Chennai, TN, India-600089. *Corresponding Author

Department of Conservative Dentistry and Endodontics, SRM Dental College, SRM Institute of Science and Technology, Ramapuram Campus, Bharathi Salai, Ramapuram, Chennai, TN, India-600089.

Department of Conservative Dentistry and Endodontics, SRM Dental College, SRM Institute of Science and Technology, Ramapuram Campus, Bharathi Salai, Ramapuram, Chennai, TN, India-600089.

\section{ABSTRACT}

Nanotechnology has brought revolutionary changes in the fields of medicine and dentistry. The application of nanoparticles in dentistry is termed as nanodentistry. Researchers worldwide experiment and employ suitable nanoparticles to overcome the existing drawbacks associated with various materials and techniques used in dentistry. Dentistry is finely segregated into various disciplines, yet still closely interwoven and interdependent to achieve a successful treatment outcome. This approach forms the backbone of interdisciplinary dentistry. From combating infections caused by microbes to improving anchorage of implants, nanotechnology and nanomaterials find multitude of use in interdisciplinary dentistry. This review article summaries the plethora of nanoparticles and their application in interdisciplinary dentistry.

\section{KEYWORDS}

Interdisciplinary Dentistry, Nanomaterials, Nanoparticles, Nanotechnology.

\section{INTRODUCTION}

The word 'Nano' has its origin from the Greek word 'dwarf'. The theory of nanotechnology was first developed in 1959 by Richard Feynman, a Nobel Prize-winning physicist, in a lecture titled, "There's plenty of room at the bottom" presented at the American Physical Society meeting held at the California Institute of Technology (CalTech) on December 29, 1959 [1]. Feynman explained that the entire area on the head of a pin (1/16 in.), if amplified 25,000 times, would have an area capable of housing all the pages of the Encyclopedia Britannica. He aptly predicted that this would be the future of material science [2]

Particles, which measure less than $100 \mathrm{~nm}$ in at least one dimension, are called as nanoparticles. Nanoparticles have distinctive advantage over their microscale or 'bulk' counterpacts, on account of their numerous advantageous properties like smaller size, greater surface energy and higher proportion of surface atoms. These materials have revolutionized the fields of engineering and medicine. They are steadily gaining foothold into dental material science and transforming clinical dentistry for the better [3]. Interdisciplinary dentistry is the key to successful treatment outcome in today's practice. In this aspect, endodontic-periodontic, endodontic-prosthodontic and endodonticperiodontic-prosthodontic interrelationship assumes significant importance. Various nanomaterials are being extensively researched and used in interdisciplinary dental practice. This article presents an overview of such materials and their usage.

\section{Nanomaterial}

A material can be called as 'Nano' when it contains components which measure less than $100 \mathrm{~nm}$ in at least one dimension. These may consist of grains, atom clusters, fibres, films and composites [3]. Nanomaterials possess unique properties due to their increased surface area and quantum effects [4]. The following nanomaterials hold key to the future of nanoscience-fuelled interdisciplinary dentistry.

Materials Of Significance In Endodontic-periodontic Interdisciplinary Approach

Chitosan Nanoparticles (CS-NPs)

Chitosan nanoparticles (CS-NPs), due to their charge and size, possess enhanced antibacterial activity [5]. Rabea et al. proposed that chitosan exerts its antibacterial effect by inhibiting enzyme activities essential for bacterial cell survival [6]. Shrestha et al. studied the effectiveness and long-term potency of CS-NPs and zinc oxide NPs ( $\mathrm{ZnO}-\mathrm{NPs}$ ) in eradicating biofilm bacteria. Both CS-NPs and ZnO-NPs were found to possess antibacterial properties even after aging for a period of 90 days. Enterococcus faecalis in planktonic and biofilm forms were tested in this study. While planktonic bacteria were completely eliminated, biofilms managed to survive even after $72 \mathrm{~h}$ of exposure to CS-NPs [7].

Sireesha et al. compared radicular dentinal tubular penetration and fracture resistance of micron- and nanoforms of chitosan and calcium hydroxide as intracanal medicaments. The authors observed that both the nanoforms exhibited superior antibacterial efficacy against Enterococcus faecalis and improved depth of penetration into the root dentin. The reduction in fracture resistance of teeth noticed with the nanoforms at 1-month interval was lesser than that observed with conventional calcium hydroxide. Hence, these nanoforms were recommended for endodontic disinfection [8]. Figures 1 and 2 show nanoforms of chitosan and calcium hydroxide with their respective scanning electron microscopic (SEM) images.

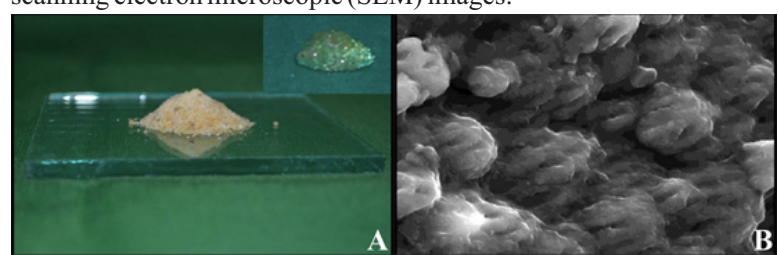

Figure 1: Nanochitosan Powder (a) And Paste (inset) Seen As Agglomerated Particles Under SEM (B).

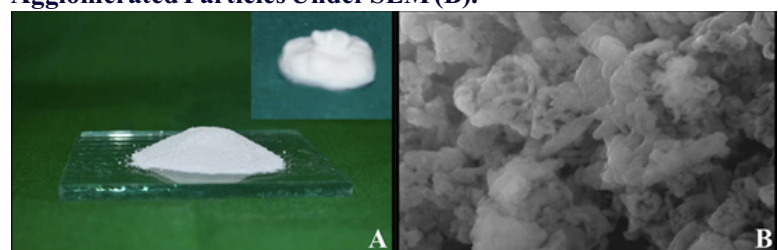

Figure 2: Nanocalcium Hydroxide Powder (a) And Paste (inset) Seen As Agglomerated Particles Under SEM (B).

Gonzalez et al. reported that copper nanoparticles/chitosan nanocomposites effectively inhibit the growth of $A$. Actinomycetemcomitans. This combination holds promise as a therapeutic agent in the augmentation of localized periodontal 
therapies [9]. Dung et al. investigated the suitability of chitosan nanoparticles as a matrix for the controlled release of antisense oligonucleotides aimed at the treatment of oral inflammatory disorders. The oligonucleotides (ONT) were mixed with chitosan, resulting in the formation of complex chitosan-oligonucleotide $(\mathrm{CH}-$ ONT) solution. Tripolyphosphate (TPP) was added as a cross linker either to chitosan or to $\mathrm{CH}-\mathrm{ONT}$ complex. The results of this study showed that the low molecular weight chitosan nanoparticles promoted prolonged release of ONT. The authors suggested that chitosan could also prevent enzymatic degradation of ONT that would possibly occur in the oral mucosa and periodontal pockets [10].

\section{Biosynthesized Silver Nanoparticles (Biosynthesized Ag-NPs)}

Silver nanoparticles, isolated and biosynthesized from the leaves of fungi, Withania somnifera were evaluated for antimicrobial efficacy against $P$. gingivalis, $B$. pumilus and $E$. faecalis. The results showed that these particles had effective antibacterial efficacy and are applicable for treating diseases of endodontic, periodontal or combined origin [11]

\section{Polydopamine Nanoparticles (PDA-NPs)}

Oxidative stress plays a major role in the induction of periodontal diseases. Bao et al. developed biocompatible polydopamine nanoparticles that could serve as potent ROS scavengers. The results showed that PDA-NPs possess antioxidative activities against toxic ROS, highlighting their potential as efficient ROS scavengers which could protect human gingival epithelial cells against oxidative stress and inflammatory reaction [12]. Deng et al. synthesized PDA-NPs incorporated peptide-decorated polycaprolactone (PCL) hybrid fibrous membrane and checked for human mesenchymal stem cell (hMSCs) adhesion, proliferation and differentiation both in vitro and in vivo conditions using mouse calvarial critical size defect model. The authors observed that PDA-NPs-PCL hybrid fibrous membrane exhibited excellent hMSC differentiation, increased apatite deposition and osteo differentiation potential without any added growth factors. Interestingly, in vivo assessment of mouse defect model showed remarkable bone reconstruction and regeneration. This could potentially aid in guided tissue regeneration for bony defects [13].

\section{Hydroxyapatite Nanoparticles (HAp-NPs)}

Sun et al. reported that HAp-NPs (or nano-HA) promoted proliferation and osteogenic differentiation of periodontal ligament cells compared to dense HAp [14]. HAp-NPs possess striking similarity in structure and chemical composition to natural teeth. Hence, they are widely used in repairing damaged enamel [15]. Li et al. and Huang et al. demonstrated that HAp-NPs can effectively remineralize artificial enamel caries lesion. Their remineralizing potential was found to be comparable to that of fluoride [16].HAp-NPs are found to be effective in the treatment of dentinal hypersensitivity (DH). Their smaller size enables easy penetration, accumulation and occlusion of the dentinal tubules, thereby reducing DH [17]. The effect of nano-HA on enamel remineralization and their influence on the sealing ability and shear bond strength of pit and fissure sealants was studied by Memarpour $e t$ $a l$. The results of the study showed that nano-HA remineralized the demineralized enamel in pits and fissures and enhanced its bond to the sealant, without compromising the sealing ability of the latter [18]. HAp-NPs have also been used as a nanocarrier for antibiotic and anticancer drugs $[19,20]$. Figure 3 shows nanohydroxyapatite particles along with their SEM and transmission electron microscopic (TEM) images.

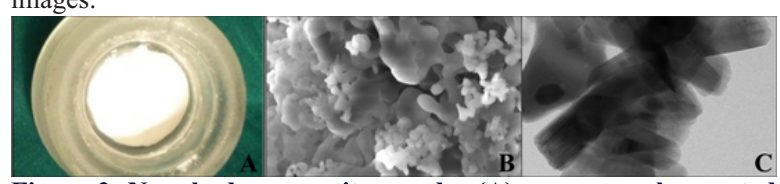

Figure 3: Nanohydroxyapatite powder (A) seen as agglomerated rods under SEM (B) and as rod shaped crystals under TEM (C).

Madhumati et al. reported that tetracycline loaded calcium deficient hydroxyapatite (TC-CDHA) nanocarriers were effective against $S$. aureus and $E$. coli bacteria. They proved the biocompatibility of these particles on human periodontal ligament cells. Augmented cellular proliferation and bone regeneration noted with this new material makes it an ideal drug delivery agent for use in periodontal therapy [21]. Similarly, Nagarathinam et al. reported that triple antibiotic paste (TAP) loaded apatitic nanocarriers (TAAN) exhibited significantly higher antibiofilm activity against $E$. faecalis compared to TAP. The authors observed an improved disinfection of dentinal tubules with
TAAN and thus, recommended its use as an intracanal medicament for root canals [22].

\section{Magnetic Nanoparticles}

Chlorhexidine (CHX) finds use in endodontics as well as in the prevention and treatment of gingivitis and periodontitis [23,24]. The fact that CHX can be rendered inactive upon its interaction with body fluids resulting in loss of its antibacterial potential lead researchers to downsize the medicament to nanomeric form $[25,26]$. Tokajuk et al. developed nanosystems comprising of amino silane-coated magnetic nanoparticles functionalized with chlorhexidine (MNP-CHX) [27] The drawbacks associated with the conventional $\mathrm{CHX}$ were overcome with the use of MNP-CHX NPs, which were found to be lethal to bacteria and fungi even in the presence of saliva. They had a profound antibacterial and antifungal effect and were found to be lethal to planktonic and biofilm-forming microorganisms. They were also capable of inhibiting the growth of multi-species biofilms.

\section{Poly (d, l-lactide-co-glycolide) (PLG) nanoparticles}

PLG nanoparticles (PLG-NPs) have been utilized to deliver antibacterial agents in the treatment of endodontic and periodontal infections. Moulari et al. investigated the antibacterial property of an ethyl acetate leaf extract of Harungana madagascariensis (HLE), a native African plant, by potentializing HLE with PLG nanoparticles. The in vitro bactericidal activity of HLE-PLG-NPs against bacteria implicated in dental caries and gingivitis were studied. The results showed that encapsulating HLE in PLG NPs enhanced the antibacterial effect of the former. This was attributed to the bioadhesive nature of PLG particles that enables prolonged adhesion and effectiveness of the antibacterial substance against the pathogens [28].

\section{Materials of significance in Endodontic-Prosthodontic interdisciplinary approach \\ Silver Nanoparticles (Ag-NPs) \\ Cheng et al. evaluated the effect of incorporating Ag-NPs on the mechanical properties and biofilm formation ability of composite resin [29]. The results showed that mechanical properties of Ag-NPs composite and control group (without Ag-NPs composite) were similar, but the counts of colony forming units were lesser in Ag-NPs composite than the control group. Melo et al. reported that incorporation of Ag-NPs in a dental adhesive disrupted the formation of biofilm, without compromising bond strength [30]. Sameiei et al. observed that addition of Ag-NPs to the commonly used endodontic repair material, MTA significantly improved the antimicrobial properties of the latter [31].}

Acosta-Torres et al. reported that Ag-NPs incorporated polymethylmethacrylate (PMMA) resin showed significantly lesser adherence of candida albicans on it compared to unmodified PMMA [32]. Zhao et al. observed that bacterial adhesion was inhibited over Titanium-implants coated with Ag-NPs for a period of up to one month. This successfully prevented post-infection at early stages of implant placement [33].

\section{Graphene Nanoparticles}

A uniform crystal lattice without any vacancies makes graphene a nanoform. Thus, graphene is used to treat various bacterial biofilms [34]. Graphene/zinc oxide nanocomposite has the potential to counteract the biofilm caused by $S$. mutans. Acrylic teeth coated with graphene are used due to their cost-effectiveness, fracture resistance and low-density property [35].

\section{Carbon Nanotubes}

Carbon nanotubes (CNTs) exhibit remarkable properties owing to their unique structure such as the presence of $\mathrm{C}-\mathrm{C}$ bond and $\mathrm{sp}^{2}$ hybridization, which confers them with strong mechanical properties. Research has shown that CNTs when incorporated into commercially available implant materials such as titanium, zirconia and hydroxyapatite improve the surface charge and texture of the implants. The CNTs surface treated implants showed better cell differentiation and rapid cell adhesion between the implant surface and bone cells and also decreased the aging of the implants [36]. Akasaka et al. coated the dentin surface with CNTs solution aimed at prevention of recurrent caries under composite restoration. The authors observed that CNTs could serve as promising pre-treatment agents under composite restoration, since they were found to bond to the collagen of dentin, serves as nucleation sites for hydroxyapatite without interfering the bond strength of composite to tooth [37]. The strength and stiffness of 
composite and epoxy resin were increased by incorporation of CNTs which acted as nanofillers [38]. Reduced polymerization shrinkage and enhanced mechanical properties were seen in CNTs incorporated heat cure monomer [39].

\section{Zirconia Nanoparticles}

Zirconia nanoparticles $\left(\mathrm{ZrO}_{2}-\mathrm{NPs}\right)$ possess a significant antimicrobial property against $C$. albicans and Aspergillus niger. Gowri et al. explained that the $\mathrm{ZrO}_{2}-\mathrm{NPs}$ produce active oxygen species, that will cause an interruption of the cell membrane of microorganism and ultimately result in increased cell membrane permeability [40]. Gad et al. observed that Candida albicans adhesion to cold-cure resin was significantly reduced by the addition of $\mathrm{ZrO}_{2}-\mathrm{NPs}$ [41]

\section{Materials Of Significance In Endodontic-periodontic- prosthodontic Multidisciplinary Approach \\ Local Nano Anaesthetic}

A colloidal suspension containing millions of anaesthetic dental nanorobots would be used to induce local anaesthesia. When deposited on the gingival tissue, the nanorobots would reach the dentin and move towards the pulp via dentinal tubules, guided by chemical differentials, temperature gradients, and positional steering by a nanocomputer under the control of the dentist. On reaching the pulp, the analgesic robots may close down all sensation in the tooth. When the treatment procedure has been concluded, the nanorobots may be ordered to reestablish all sensations and to exit from the tooth. This technique will be advantageous as it would greatly reduce apprehension and be fast and totally reversible [42]. Suture needles incorporating nanosized stainless steel crystals have been developed. Nanotweezer was first created by Philip Kim and Charles Lieber at Harward University in 1999. Its working end is a pair of electrically controlled carbon nanotubes made from a bundle of multiwalled carbon nanotubes. To operate the tweezers, a voltage is applied across the electrode that develops a positive and negative electrostatic charge on the electrode [43].

\section{CONCLUSION}

Nanotechnology will bring even greater changes to the field of dentistry. Nanoparticle functionalization can result in higher drug efficacy as more bioactive molecules could be loaded onto one nanoparticle. Newer economic multifunctional nanoparticles and their modifications should be developed based on clinical requirements and human needs. The whole concept of nanotechnology in health care should be accepted with positive zeal and caution for future development

\section{REFERENCES}

1. Feynman RP. There is plenty of room at the bottom. California Institute of Technology Engineering and Science magazine. 1960;23(5):22-36. https://resolver.caltech.edu/ CaltechES:23.5.1960Bottom.

2. Bhardwaj A, Bhardwaj A, Misuriya A, Maroli S, Manjula S, Singh AK. Nanotechnology in dentistry: present and future. J Int Oral Health. 2014;6(1):121-6. PMID: 24653616; in dentistry: present an

3. Jeevanandam J, Barhoum A, Chan YS, Dufresne A, Danquah MK. Review on nanoparticles and nanostructured materials: history, sources, toxicity and regulations. Beilstein J Nanotechnol. 2018;9:1050-74. https://doi.org/10.3762/bjnano.9.98

4. Kumar N, Kumbhat S. Essentials in nanoscience and nanotechnology. 1st ed. New York: Wiley online library; 2016

Kishen A. Nanotechnology in endodontics: current and potential clinical applications. 1st ed. Switzerland: Springer; 2015.

6. Atay HY. Antibacterial activity of chitosan-based systems. In: Jana S, Jana S, editors. Functional chitosan: drug delivery and biomedical applications. $1^{\text {st }}$ ed. Switzerland: Springer; 2020. https://doi.org/10.1007/978-981-15-0263-7.

7. Shrestha A, Shi Z, Neoh KG, Kishen A. Nanoparticulates for antibiofilm treatment and effect of aging on its antibacterial activity. J Endod. 2010;36(6):1030-5. https://doi.org/10.1016/j.joen.2010.02.008.

8. Sireesha A, Jayasree R, Vidhya S, Mahalaxmi S, Sujatha V, Kumar TS. Comparative evaluation of micron- and nano- sized intracanal medicaments on penetration and fracture resistance of root dentin - an in vitro study. Int J Biol Macromol. 2017;104(Pt B):1866-73. https://doi.org/10.1016/j.ijbiomac.2017.05.126. Epub 2017 May 22.

9. González JP, Covarrubias C, Cádiz M, Corral C, Cuadra F, Fuentevilla I, et al. Design of antimicrobial release sysytems based on chitosan and copper nanoparticles for localized periodontal therapy. J Dent \& Oral Disord. 2016;2(7)1035.

10. Dung TH, Lee SR, Han SD, Kim SJ, Ju YM, Kim MS, et al. Chitosan-TPP nanoparticle as a release system of antisense oligonucleotide in the oral environment. J Nanosci Nanotechnol, 2007, 7(11),3695-9. https://doi.org/10.1166/jnn.2007.041.

11. Halkai KR, Mudda JA, Shivanna V, Rathod V, Halkai RS. Evaluation of antibacterial efficacy of biosynthezised silver nanoparticles derived from fungi against endo-perio pathogens porphyromonas gingivalis, bacillus pumilus, and enterococcus faecalis. J Conserv Dent. 2017;20(6):398-404. https://dx.doi.org/10.4103\%2FJCD.JCD 17317.

12. Bao X, Zhao J, Sun J, Hu M, Yang X. Polydopamoine nanoparticles as efficient scavangers for reactive oxygen species in periodontal diseases. ACS Nano. 2018;12(9):8882-92. https://doi.org/10.1021/acsnano.8b04022.

13. Deng Y, Yang WZ, Shi D, Wu M, Xiong XL, Chen ZG, et al. Bioinspried and osteopromotive polydopamine nanoparticle-incorporated fibrous membaranes for robust bone regeneration. NPG Asia Materials. 2019;11(39):1-13. https://oi.org/10.1038/s41427-019-0139-5.

14. Sun W, Chu C, Wang J, Zhao H. Comparison of periodontal ligament cells responses to dense and nanophase hydroxyapatite. J Mater Sci Mater Med. 2007;18(5):677-683. https://doi.org/10.1007/s10856-006-0019-8.

15. Pokrowiecki R, Palka K, Mielczarek A. Nanomaterials in dentistry: a cornerstone or a black box? Nanomedicine (Lond). 2018;13(6):639-67. https://doi.org/10.2217/nnm2017-0329

16. Manchery N, John J, Nagappan N, Subbiah GK, Premnath P. Remineralization potentia of dentifrice containing nanohydroxyapatite on artificial carious lesions of enamel: A comparative in vitro study. Dent Res J (Isfahan). 2019;16(5):310-7. PMID: 31543937 PMCID: PMC6749857

17. Khetawat S, Lodha S. Nanotechnology (nanohydroxyapatite crystals): recent advancement in treatment of dentinal hypersensitivity. Journal of Interdisciplinary Medicine and Dental Science. 2015;3:181. https://doi.org/10.4172/2376032X.1000181.

18. Memarpour M, Shafiei F, Rafiee A, Soltani M, Dashti MH. Effect of hydroxyapatite nanoparticles on enamel remineralization and estimation of fissure sealant bond strength to remineralized tooth surfaces: an in vitro study. BMC Oral Health. 2019;19(1):92. https://doi.org/10.1186/s12903-019-0785-6.

19. Simon AT, Dutta D, Chattopadhyay A, Ghosh SS. Copper nanocluster-doped luminescent hydroxyapatite nanoparticles for antibacterial and antibiofilm applications. ACS Omega. 2019;4(3):4697-706. https://doi.org/10.1021/acsomega.8b03076.

20. Venkatasubbu GD, Ramasamy S, Reddy GP, Kumar J. In vitro and in vivo anticancer activity of surface modified paclitaxel attached hydroxyapatite and titanium dioxide nanoparticles. Biomed Microdevices 2013;15(4):711-26. $\mathrm{n}$ an op articles. B i m e d M i r o de
https://doi.org/10.1007/s10544-013-9767-7.

21. Madhumathi K, Sampath Kumar TS. Regenerative potential and anti-bacterial acitvity of tetracycline loaded apatitic nanocarriers for the treatment of periodontitis. Biomed Mater. 2014;9(3):035002. https://doi.org/10.1088/1748-6041/9/3/035002.

22. Nagarathinam S, Sujatha V, Madhumathi K, Mahalaxmi S, Vanajassun PP, Sampath Kumar TS. Effect of triple antibiotic loaded apatitic nanocarriers on enterococcus faecalis biofilm - an in vitro study. Journal of Drug Delivery Science and Technology, 2019;51:499-505. https://doi.org/10.1016/j.jddst.2019.03.035.

23. Manikandan D, Balaji VR, Niazi TM, Rohini G, Karthikeyan B, Jesudoss P. Chlorhexidine varnish implemented tretament strategy for chronic periodontitis: a clinical and microbial study. J Pharm Bioallied Sci. 2016;8(Suppl 1):S133-7. https://dx.doi.org/10.4103\%2F0975-7406.191943.

24. Supranoto SC, Slot DE, Addy M, Van der Weijden GA. The effect of chlorhexidine dentrifice or gel versus chlorhexidine mouthwash on plaque, gingivitis, bleeding and tooth discoloration: a systematic review. Int J Dent Hyg. 2015;13(2):83-92 https://doi.org/10.1111/idh.12078

25. Brooks SE, Walczak MA, Hameed R, Coonan P. Chlorhexidine resistance in antibioticresistant bacteria isolated from the surfaces of dispensers of soap containing chlorhexidine. Infect Control Hosp Epidemiol, 2002;23(11):692-5. https://doi.org/10.1086/501996.

26. Liu Q, Zhao H, Han L, Shu W, Wu Q, Ni Y. Frequency of biocide-resistant genes and suspectibility to chlorhexidine in high-level mupirocin-resistant, methicillin-resistan staphylococcus aureus (MuH MRSA). Diagn Microbiol Infect Dis. 2015;82(4):278-83. https://doi.org/10.1016/j.diagmicrobio.2015.03.023.

27. Tokajuk G, Niemirowicz K, Deptula P, Piktel E, Cieśluk M, Wilczewska A. Use of magnetic nanoparticles as a drug delivery system to improve chlorhexidine antimicrobial activity. Int J Nanomedicine. 2017;12:7833-46. https://dx.doi.org/10.2147\%2FIJN.S140661.

28. Moulari B, Lboutounne H, Chaumont JP, Guillaume Y, Millet J, Pellequer Y Potentiation of the bacterial activity of harungana madagascariensis lam. Ex poir (hypericaceae) leaf extract against oral bacteria using poly (D, L-lactide-co-glycolide) nanoparticles: in vitro study. Acta odontol Scand. 2006;64(3):153-8. https://doi.org/10.1080/00016350500483152.

29. Cheng L, Weir MD, Xu HHK, Antonucci JM, Lin NJ, Lin-Gibson S. Effect of amorphous calcium phosphate and silver nanocomposites on dental plaque microcosm biofilm. J Biomed Mater Res B Appl Biomater. 2012;100(5):1378-86 https://doi.org/10.1002/jbm.b.32709.

30. Melo MA, Cheng L, Zhang K, Weir MD, Rodrigues LK, Xu HH. Novel dental adhesives containing nanoparticles of silver and amorphous calcium phosphate. Dent Mater. 2013;29(2):199-210. https://doi.org/10.1016/j.dental.2012.10.005.

31. Sameiei M, Aghazadeh M, Lotfi M, Shakoei S, Aghazadeh Z, Vahid Pakdel SM. Antimicrobial efficay of mineral trioxide aggregate with and without silver nanoparticles. Iran Endod J. 2013:8(4):166-70. PMCID: PMC3808675 PMID: 24171023

32. Acosta-Torres LS, Mendieta I, Nuñez-Anita RE, Caiero-Juárez M, Castaño VM Cytocompatble antifungal acrylic resin containing silver nanoparticles for denture. Int $J$ Nanomedicine. 2012;7:4777-86. https://doi.org/10.2147/ijn.s32391

33. Zhao L, Wang H, Huo K, Cui L, Zhang W, Ni H. Antibacterial nano-structured titania coating incorporated with silver nanoparticles. Biomaterials. 2011;32(24):5706-16. https://doi.org/10.1016/j.biomaterials.2011.04.040

34. Mejías Carpio IE, Santos CM, Wei X, Rodrigues DF. Toxicity of a polymer-graphene oxide composite against bacterial planktonic cells, biofilms, and mammalian cells. Nanoscale. 2012;4(15):4746-56. https://doi.org/10.1039/c2nr30774j.

35. Kulshrestha S, Khan S, Meena R, Singh BR, Khan AU. A graphene/zinc oxide nanocomposite film protects dental implant surfaces against cariogenic streptococcus mutans. Biofouling, 2014;30(10):1281-94. https://doi.org/10.1080/08927014 2014.983093.

36. Teh SJ, Lai CW. Carbon nanotubes for dental implants. In: Asiri A, Inamuddin, Mohammad A, editors. Applications of Nanocomposite Materials in Dentistry. 1st ed. Duxford: Woodhead Publishing; 2019.

37. Akasaka T, Nakata K, Uo M, Watari F. Modification of the dentin surface by usin carbon nanotubes. Biomed Mater Eng. 2009;19(2-3):179-85. https://doi.org/10. 3233/bme-2009-0578.

38. Baudot C, Tan C, Wang C. Nano-tailoring of carbon nanotube as nano-fillers for composite materials applications. $2^{\text {nd }}$ IEEE International Nanoelectronics Conference Proceedings. 2008; 570-72

39. Sree L, Balasubramanian, Deepa. Nanotechnology in dentistry - A review. Internantional Journal of Dental Sciences and Research. 2013;1(2):40-4 https://doi.org/ 10.12691/ijdsr-1-2-4

40. Gowri S, Gandhi RR, Sundrarajan M. Structural, optical, antibacterial and antifungal properties of zirconia nanoparticles by biobased protocol. J Mater Sci Technol. 2014;30(8):782-90. https://doi.org/10.1016/j.jmst.2014.03.002.

41. Gad MM, Al-Thobity AM, Shahin SY, Alsaqer BT, Ali AA. Inhibitory effect of zirconium oxide nanoparticles on candida albicans adhesion to repaired polymethyl methacrylate denture bases and interim removable prostheses: a new approach for denture stomatitis prevention. Int J Nanomedicine. 2017;12:5409-19. https://doi.org/10.2147/ijn.s142857

42. Srivastava S, Bhalla VK. Nanotechnology in interdisciplinary dentistry. Internationa Journal of Scientific Research 2020; 9(1):55-6. https://www.doi.org/10.36106/ijsr.

43. Markan S, Lehl G, Kapoor S. Recent advances of nanotechnology in endodontics, conservative and preventive dentistry -A review. J Dent Oral Biol. 2017;2(10):1067. 